\title{
Architecture and key technologies for Internet of Vehicles: a survey
}

\author{
Fangchun Yang, Jinglin Li*, Tao Lei, Shangguang Wang \\ State Key Laboratory of Networking and Switching Technology, Beijing University of Posts and \\ Telecommunications, Beijing 100876, China \\ * Corresponding author, Email: jlli@bupt.edu.cn
}

\begin{abstract}
In recent years, IoV (Internet of Vehicles) has become one of the most active research fields in network and intelligent transportation system. As an open converged network, IoV plays an important role in solving various driving and traffic problems by advanced information and communications technology. We review the existing notions of IoV from different perspectives. Then, we provide our notion from a network point of view and propose a novel IoV architecture with four layers. Particularly, a novel layer named coordinative computing control layer is separated from the application layer. The novel layer is used for solving the coordinative computing and control problems for human-vehicle-environment. After summarizing the key technologies in IoV architecture, we construct a VV (Virtual Vehicle), which is an integrated image of driver and vehicle in networks. VVs can interact with each other in cyber space by providing traffic service and sharing sensing data coordinately, which can solve the communication bottleneck in physical space. Finally, an extended IoV architecture based on VVs is proposed.
\end{abstract}

Keywords: Internet of Vehicles, converged network, virtual vehicle, application service

Citation: F. H. Yang, J. L. Li, T. Lei, et al. Architecture and key technologies for Internet of Vehicles: a survey [J]. Journal of communications and information networks, 2017, 2(2): 1-17.

\section{Introduction}

With the rapid development of social economy and the process of industrialization, the number of vehicles has been increasing dramatically in cities. However, owing to limited capacity, roads become saturated, which causes considerable number of issues, such as traffic congestion, traffic accident, energy consumption, and environmental pollution.

In the process of exploring solutions to the abovementioned issues, the automotive industry introduces new energy and energy-saving vehicles to achieve the coordinated development of automotive industry and environment. In addition, it is essential to fully use the information and communication technologies for achieving the coordinated development of human, vehicle, and environment, which can alleviate traffic congestion, enhance transportation efficiency, and enhance existing road capacity. $\mathrm{IoV}^{[1]}$ emerges and quickly becomes the research focus both in academia and industry. These studies predominantly concentrate on intelligent transportation and Telematics (telecommunications and informatics). The intelligent transportation studies predominantly focus on the enhancement of human travel efficiency and travel safety, such as the United States

Manuscript received Jan. 16, 2017; accepted Apr. 01, 2017

This work is supported by the National Science and Technology Major Project of China (No. 2016ZX03001025-003), Special Fund for Beijing Common Construction Project. 
project Intelligent vehicle-road system, the Europeans plan Eureka and Japans Advanced dynamic traffic in-formation system. As for Telematics studies, OnStar is an earlier one ${ }^{[2]}$. Similar to the current Apples CarPlay car system, Googles Android Auto platform $^{[3,4]}$, Baidus CarLife systems, and LeTVs car open platform, OnStar predominantly utilizes mobile communication technology and GPS (Global Positioning System) for providing perfect wireless services for vehicles ${ }^{[5]}$. As a derivative of the IoT (Internet of Things) and mobile Internet applications in transportation, IoV is a blend of mobile Internet, ITS (Intelligent Transportation System), cloud computing, car electronics, intelligent transportation technology and geographic information systems. The industrial chain of IoV includes the automobile manufacturing and services, communications and information services, transportation, finance, insurance services and public services, and other fields. The long industrial chain generates different understandings of IoV, which result in different understandings of the connotation and the architecture in the academia and industry.

Owing to the various unsolved issues, it is significantly challenging to implement IoV. The predominant challenges are described as follows.

(i) Inconsistent understanding of IoV hinders its development. Owing to the different people understanding differently, people from different fields propose different concepts and architectures of IoV. However, these differences lead to several problems, such as human and vehicle coordination problem, which will impede the development of IoV.

(ii) Human, vehicle, and environment should be integrated deeply in IoV. With the development of the intelligent vehicle, the vehicles have become an extension of the human perception. Hence, the human, vehicle, and environment integration becomes the core of IoV service object.

(iii) The coordination behaviors between human and vehicle are considerably complex. Because the vehicle movement behaviors exhibit an impact on the human decisions, the intelligent vehicles cannot simply act as human and make decisions. Human and vehicles should coordinate to make decisions. The IoV application scenarios are spatiotemporally diverse, which leads to the complexity of human and vehicle coordination.

To overcome the above challenges, this paper first focuses on the concept and architecture of IoV. In this part, the definition of IoV is provided and an architecture of IoV based on the functional requirements and expected goals are proposed. The architecture includes the environment sensing and control layer, network access and transport layer, coordinative computing control layer, and application layer. Second, we review and summarize the core technologies of each layer in the IoV architecture. Particularly, in the environment sensing and control layer, we introduce vehicle control and environment sensing technology, respectively. As for network access and transport layer, we introduce the current mainstream of vehicular wireless network access technology and heterogeneous network transmission control technology. In the coordinative computing control layer, we mainly introduce the current studies of coordination among human-vehicle-environment in IoV. In the IoV application layer, the closed services and open services are reviewed. Third, according to the research status of architecture and key technologies, we summarize the existing problems and challenges. Meanwhile, we propose to construct an image, VV, in coordinative computing control layer. In addition, the concept and architecture of a VV are provided. Finally, we discuss the future research directions of IoV based on VVs.

\section{Concept and architecture of IoV}

\subsection{Concept of IoV}

As an important branch of IoT in the transportation field, IoV covers a wide range of technologies and applications, including intelligent transportation, vehicular information service, modern information and communications technology, and automotive electronics. However, owing to different understandings of the connotation of IoV in various research fields, 
there is no uniform definition of IoV. Some related definitions are provided in the following.

$\mathrm{IoV}$ is considered as a vehicle connected network based on vehicular information system. Drivers are informed of the status of vehicle based on outside information sensed by vehicle electronic equipment. Here, the essence of the IoV study is based on drivers. ITS realizes the traffic guidance and control through the interconnection between vehicle and vehicle, and vehicle and the environment, which essentially studies IoV from the perspective of traffic. The information and communication system regards vehicle as a mobile terminal from the perspective of industrial chain, which uses the communication network, data platform, and supported service platform. The vehicles establish connections to create a network, namely the networking vehicle. It essentially studies IoV from the perspective of the communication network.

Alam et al. ${ }^{[3]}$ regard IoV as an ITS integrated IoT from the perspective of intelligent transportation. In other words, IoV is the vehicular ITS, which regards the driving vehicle as the information sensing object. IoV integrates the intelligent sensors, wireless communication technology, distributed data, information processing, and Internet technology for achieving the efficient exchange of information and sharing between human and vehicle, vehicle and vehicle, and vehicle and roadside infrastructure. Hence, IoV improves road traffic conditions and travel efficiency by realizing the intelligent control among human, vehicles, roads, and transportation facilities. In addition, IoV extends the scope of integrated information services and intelligence decision-making system, which can be used for solving the traffic information sensing and processing problems.

From the perspective of network interconnection, Hartenstein et al. ${ }^{[6]}$ consider that IoV is a network that integrates in-vehicle network, inter-vehicles network, and vehicular mobile Internet. They found that RFID (Radio Frequency Identification), GPS, mobile communication, wireless network, and network service support technology in IoV are used for achieving the real-time wireless communication and information exchange system network between vehicle and road, vehicle and vehicle, vehicle and human, and vehicle and cities. Moreover, it achieves the intelligent traffic management, intelligent dynamic information services, and vehicle intelligent control. It is a typical application of ICT (Information and Communications Technology) in the field of transportation systems, solving the interconnection problem between vehicle and vehicle, and vehicle and network.

$\mathrm{Li}^{[7]}$ presents the definition of $\mathrm{IoV}$ from the perspective of integration of on-board sensors and communication technology. They consider IoV as vehicles carrying advanced sensors, controllers, actuators, and other devices, which integrate modern communications and network technology for providing the vehicles complex environmental sensing, intelligence decision-making, and control functions. According to this definition, the vehicles in IoV can fulfill safety, energy saving, and comfortable driving. Hence, IoV, from this perspective, predominantly solves the problem of intelligent decision-making of vehicles.

It can be observed that there are differences in the definitions of IoV when people provide their definition in view of their own professional areas. Vehicle enterprises predominantly focus on vehicular information system, and regard IoV as the platform achieving vehicle informatization and intelligence. The transportation holds the view around the intelligent transportation, and regards IoV as a mode for enhancing the level of intelligent transportation technology. The Internet regards $\mathrm{IoV}$ as a type of mobile terminal for mining new services. Nowadays, the mobile Internet increasingly penetrates and invades all walks of life, and the vehicle will no longer be a simple technique of transport. It will become an increasingly open platform, which integrates hightech, Internet, and intelligence. Therefore, the development of IoV will certainly become the core of cross-border integration, and all areas will also be integrated into the IoV.

Moreover, we provide the definition of $\mathrm{IoV}$ as follows ${ }^{[8]}$ : IoV is an open converged network sys- 
tem based on multihuman, multimachine, multivehicle, and environment coordination. This system is controllable, manageable, operational, and trustable. It senses, recognizes, transmits, and computes the large-scale complex static/dynamic information of human, vehicle, network communication, and road traffic infrastructure, using advanced information communication and processing technology. The information provided above is used for solving the problem of computability, scalability, and sustainability of intelligent management and information service in the heterogeneous mobile converged network environment. Finally, it helps to realize the deep integration of human, vehicle, and environment.

\subsection{Architecture of IoV}

Currently, there is no specific architecture of IoV because of the existing different comprehension of IoV. Some researchers propose the architecture of IoV based on IoT, which is similar as IoV and consists of sensing layer, network layer, and application layer. However, IoV is not only a service network for vehicle-to-vehicle communication or vehicle terminals, but also a complex system that has the feature of human-vehicle-environment tightly coordinative interaction and highly dynamic evolution. In the process of cooperation among human, vehicle, and environment, IoV is required for supporting pervasive computing, cognitive computing, and new types of information computing, which requires novel requirements on architecture and support capacity for IoV. To achieve this target, we integrate the architectures of IoV proposed by current academia and industry, and divide it into four layers, as shown in Fig. 1, that is, vehicle network environment sensing and control layer, network access and transport layer, coordinative computing control layer, and application layer.

(1) Vehicle network environment sensing and control layer

The environment sensing is the recognition basis for IoV services, such as services of autonomous vehicle, intelligent traffic, and vehicle information. The control of vehicle and the traffic environment object are the basis of IoV services implementation. From the perspective of vehicles, they sense environment information around themselves via autopilot system, traffic jam auxiliary system, and sensor system for achieving auxiliary driving. In terms of environment, this layer studies the procedure to monitor and extract various dynamic information of human, vehicles, and environment through sensing technology. In addition, it focuses on the procedure to receive and execute coordinative control instructions and then feedback result to cooperative control. Finally, it implements the capabilities of swarm sensing in swarm model and participates sensing in individual model.

(2) Network access and transport layer

The main function of this layer is to realize the network access, data processing, data analysis, and data transmission. At the same time, it can also realize the remote monitoring and nodes management within the IoV. The basic idea of this layer is realizing the inter-connection and information exchange, which includes the access network, the transmission, and control network. The access network provides real-time, three-dimensional, and seamless heterogeneous network access for vehicles connecting network. The transmission and control network responds to dispatch access resources and balances information load. Then, it establishes a stable, qualityguaranteed information and communication transmission channel considering the network load conditions and access resource constraints.

(3) Coordination computing control layer

This layer provides IoV applications with the network-wide capability of coordinative computing and control for human-vehicle-environment, such as data processing, resource allocation, and swarm intelligence computing. From the perspective of IoV objects coordinative individual model, this layer should provide the capability of human-vehicle coordinative computing control to support the humanvehicle, which achieves coordination in the IoV environment. From the perspective of IoV objects coordinative swarm model, this layer should provide the 


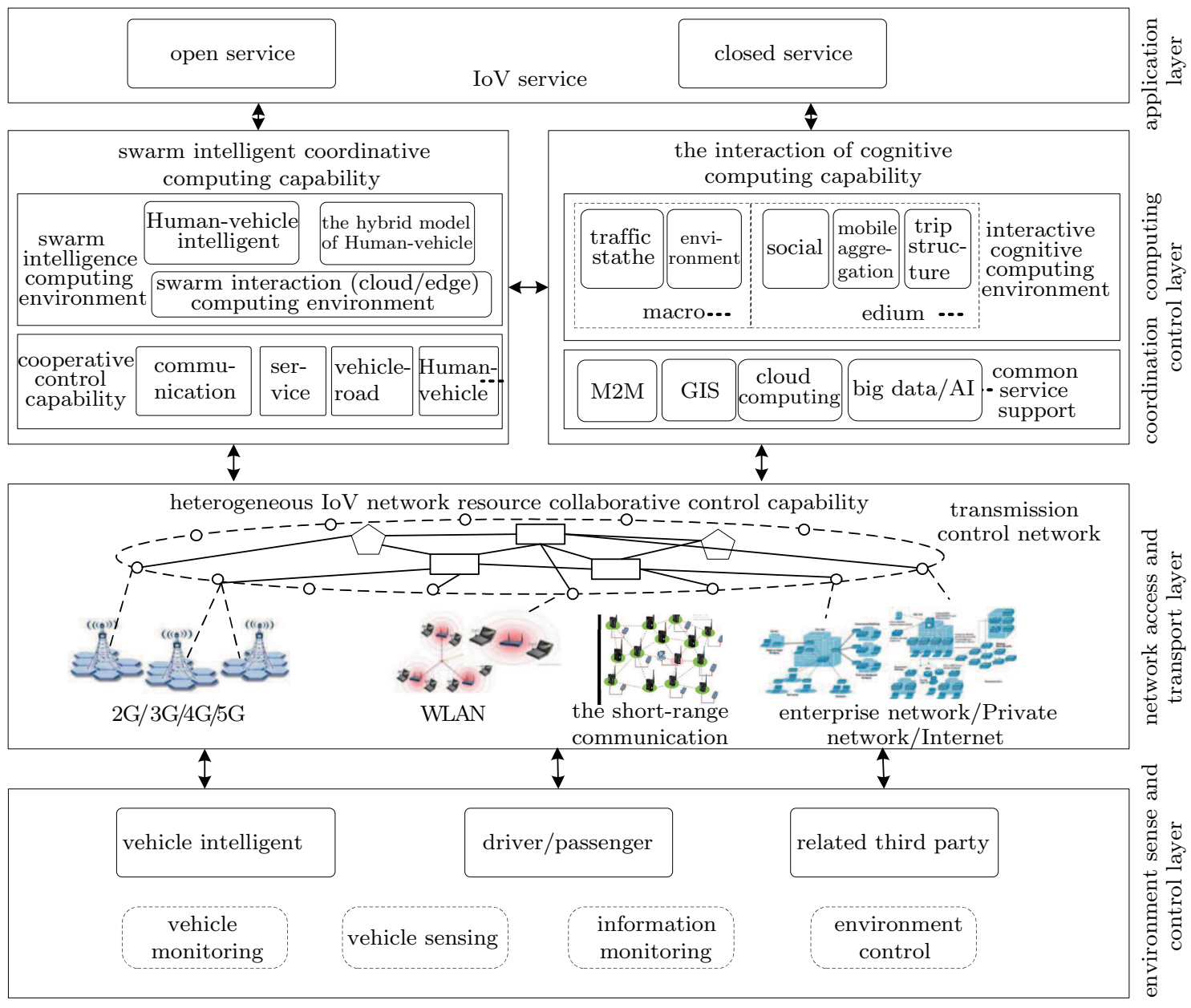

Figure 1 Architecture of IoV

capabilities, including the multihuman and multivehicle coordinative computing control and service coordinative management, for the purpose of supporting the swarm intelligence computation and various services. In addition, in order to meet the coordinative control requirements, this layer should also provide the capability of communication coordinated management.

(4) Application layer

The application layer of IoV provides various types of services to achieve the requirements of humanvehicle-environment coordination services. In addition, the application layer should be in the open state and should share information to support the novel service and business operating model. Owing to the closed status quo of the application, the application layer can be classified into closed services and open services. The closed services are closely related to the specific industry applications, such as the intelligent traffic command and control platform. The open services cover various existing open applications, such as real-time traffic service provided by various Internet service providers. The application layer should also provide open service capabilities to third party providers.

\section{Vehicle network environment sens- ing and control layer}

\subsection{Vehicle network environment sens- ing}

To sense environment, vehicle can directly sense surroundings via its own sensors. On the other hand, 
one can indirectly perceive surroundings by sharing information. As more vehicle electronic equipment is configured, all on-board sensors can be connected by in-vehicle network to create a vehicular sensing system. This system can support the perception ability, which can sense human in vehicle, vehicle itself, and the outside environment. Since the driver assistance is provided, this system belongs to direct perception. Moreover, vehicles compose a swarm of environmental perception to sense environment coordinately, which belongs to the indirect perception.

(1) Direct perception

On-board sensor system uses the camera, laser radar, ultrasonic sensor, microwave radar, GPS, magnetic compass, and mileage recorder for perceiving the environment. ITS uses a variety of roadside sensors to sense the vehicle information, such as ITS using ETC (Electronic Toll Collection) and RFID for identifying vehicles. At present, the sensor applications based on automated driving can be basically classified into three categories: the application of acceleration sensor, gyroscope, magnetic induction sensor; the application based on intelligent mobile phone or camera; and the application based on radar sensor.

At present, accelerometer, gyroscope, and magnetic induction sensor are the most common sensors (usually these three sensors can achieve safe driving $\left.{ }^{[9]}\right)$ in mobile devices, including the vehicle and mobile phone. They typically use these three types of sensors for describing the personalization behavior. The acceleration sensor can measure the instantaneous acceleration of the vehicle. Therefore, it can be used for describing the trajectory of the vehicle. The gyroscope is very sensitive to rotation, which can measure the rotating angular velocity. Therefore, it can be used for describing the vehicle turning movements. Moreover, the magnetic sensors can record the driving direction of the vehicle.

Camera adopts the human visual method to capture environment information. To capture the driver behaviors and model these behaviors in 3D environment ${ }^{[10]}$, the camera can not only provide driverless experimental verification, but also capture the context information, which is related to driving behavior (such as facial expression and head movement, vehicle operation). Monitoring the changes of traffic lights using camera, the driver can be suggested to a reasonable speed, which can reduce fuel consumption and carbon dioxide emission caused by the frequent stop and go. At the same time, it also can ease the depression of drivers caused by congestion $^{[11]}$.

The radar sensor can increase the ability of the environmental perception of vehicles. For example, single line laser radar scanner can be used for probing the road obstacle, and the panoramic laser radar can be used for sensing the overall environment, detect the dynamic obstacle, identify the intersection, and build map. At present, the Google self-driving vehicle ${ }^{[12]}$ and Baidu self-driving vehicle are equipped with panoramic laser radar, which have 64 laser rangefinder. It can obtain a complete frame with scanning one revolution to ensure the real-time detection of the road environment. However, in the actual market, it is a difficult problem to persuade the driver to install these expensive sensors. As a result, the applications of external sensors are mostly used for self-driving vehicle experiments.

(2) Indirect perception

Indirect perception is mentioned as obtaining information from others via communicating with each other. For example, vehicles in VANET (Vehicular Ad hoc NETworks) exchange information and send traffic information through network. From the point of individual coordination model in IoV, various sensing devices create an on-board sensor system. This system provides the ability to sense human in vehicle, vehicle, and environment. It can also realize the indirect perception via car networks, inter-vehicle networks, and mobile Internet. For example, driverless vehicle is a typical system that linked various sensing devices with the vehicle. All devices in the driverless ones sense the data of vehicle itself and the surrounding environment coordinately. Additionally, they can provide the data to driverless vehicle for decision making ${ }^{[12]}$. Besides, indirect per- 
ception can be used in road detection. For example, Zhou et al. ${ }^{[13]}$ detect the road with sensing visual and spatial information, which are sensed by various sensors. Alvarez et al. ${ }^{[14]}$ use a shadow-invariant feature space combined with a model-based classifier for implementing vision-based road detection.

From the perspective of objects coordination model in IoV, each perception individual in $\mathrm{IoV}$, such as mobile terminal and roadside infrastructure, can form a swarm to sense coordinately. For instance, the traffic information sensing requires the coordination of different sensing individuals. In the sensing process, the sensing individual adopts vary sensing models according to the different sensed objects, such as fixed infrastructure, mobile devices, and swarm behavior. Then, all sensing information is mixed effectively using a unified structure of the system for providing the traffic information. For example, the traffic flow can be used for predicting congestion $^{[15]}$. Santini et al. ${ }^{[16]}$ propose an approach that uses vehicles coordination in a swarm to sense the safe distance information.

The present sensing approaches for vehicles always focus on one aspect of IoV. However, there is no such procedure to combine all sensing technologies effectively for sensing data coordinately in IoV.

\subsection{Vehicle control}

Auxiliary driving is invariantly a hotspot, which focuses on the automotive control in the traditional automobile manufacturers. Driverless vehicle is the ultimate of auxiliary driving, which is classified into five grades. The 0th grade is that the vehicle is controlled by the driver at all time. The first grade is that in which a single function of automation vehicle is realized. The second grade is that, in the vehicle, at least two functions can be controlled automatically. The third grade is that the vehicle can operate by itself under some conditions without driver control, and it can give sufficient time for driver to control the vehicle when it is necessary. The fourth grade is that the vehicle can complete all safety control without driver participation in the entire jour- ney, including parking and control empty.

The driverless vehicles predominantly utilize the on-board sensors to sense the state of vehicle itself and the surrounding environment. According to the path, vehicle position, and obstacle information, it can control vehicle speed and steering through automatic control of the actuator ${ }^{[17,18]}$. With the development of artificial intelligence and control theory, driverless vehicles have become one of the hot spots. Carnegie Mellon Universitys Tartan Racing fleet of driverless vehicle Boss is composed of a Chevrolet Tahoe ${ }^{[19]}$, and the software system consists of four subsystems: sensing system, motion planning system, mission planning system, and behavior system ${ }^{[20]}$. In the city environment, it can realize the obstacle avoidance, obstacle avoidance, automatic parking, and other functions. Since 2010, Google Corporation has set up driverless vehicle research project ${ }^{[5,21]}$, which tries to develop an artificial intelligence system to replace manual labor/people driving. On December 14, 2015, Baidu announced to set up the driverless vehicle department. Therefore, the research of driverless vehicle can be classified into two categories in IoV: one is the vehicle control theory study based on vehicle itself, and the other is the driving behavior study.

The key techniques of driverless vehicle control theory ${ }^{[22]}$ include driving environment perception, path planning, intelligent decision, vehicle navigation, and automatic control. Vehicle control includes driving with the desired speed, performing the planning path, and following the front vehicle. Since the driverless vehicle system is a nonlinear system, designing the controller to realize the longitudinal and lateral control with high accuracy is the key problem. To solve this problem, the vehicle dynamics model and the kinematics mathematical model are invariantly used. Whitecomb and Milliken ${ }^{[23]}$ first studied the vehicle two degrees of freedom linear model, then in 1956 and 1965, Segel ${ }^{[24]}$ put forward linear three degrees of freedom and linear model and four degrees of freedom model, respectively. In 1995, Hedrick et al. ${ }^{[25]}$ from the American University of California proposed an 18 vehicle model, which describes the 
vehicle tires, engine, suspension, chassis, and other characteristics accurately.

In the research of intelligent driving behavior, $\mathrm{Li}$ et al. ${ }^{[26]}$ conducted a survey on the current intelligent behavior researches, which includes four aspects. They are the longitudinal analysis of driving behavior and collision avoidance ${ }^{[27]}$, driving behavior analysis and road horizontal departure warning ${ }^{[28]}$, complex driving behavior learning ${ }^{[29]}$, and driver status analysis ${ }^{[30]}$. Hence, the study of driverless vehicle tends to driver model from vehicle handling and stability, and tends to the performance of the entire road-vehicle-driver system from a high degree of freedom vehicle model. McRuer et al. ${ }^{[31]}$ proposed a driver model, which is applicable to the closed-loop technology of plan in vehicles to establish the PID (Proportion-Integral-Derivative) model, cross classification model, and low order prediction model based on the classic control theory.

All the above-mentioned research predominantly focuses on driving safety and auxiliary driving. They do not consider the procedure to achieve coordination control between vehicle control and traffic control, which is significantly important for IoV.

\section{Network access and transport layer}

\subsection{Network access}

At present, the network access technologies of IoV can be classified into inter-vehicle networks access technologies and mobile Internet access technologies. The access technologies of inter-vehicle networks include DSRC (Dedicated Short Range Communications) and WAVE (Wireless Access in the Vehicular Environment). Moreover, the mobile Internet access technologies include LTE (Long Term Evolution) and WiMAX-WLAN. Vehicles rely on these wireless communication technologies to access networks, which can realize the communication between vehicles and networks.

DSRC is a type of efficient wireless communication technology ${ }^{[32]}$, which supports the moving tar-

\footnotetext{
1) http://www.huawei.com/minisite/hwmbbf15/en/lte-v.html
}

get recognition and two-way communication with high-speed motion in a specific area (usually tens of meters). DSRC adopts the communication standard IEEE 802.11a. Its largest transmission distance is $1000 \mathrm{~m}$ and the default transmission rate is $6 \mathrm{Mbit} / \mathrm{s}$ (up to $27 \mathrm{Mbit} / \mathrm{s})^{[33]}$. DSRC has two operating modes: one is to establish the connection among vehicles, which is used for enhancing the traffic safety via keeping safe distance and warning traffic accidents. The other is to establish the connection between vehicle and road, which is used for easing traffic pressure via optimal route. Currently, WAVE technology has become one of the main access technologies ${ }^{[34,35]}$ to connect the network. The WAVE is used for solving channel blocking problem in the physical layer when vehicles access network ${ }^{[36]}$. Shen et al. ${ }^{[37]}$ proposed a multi-priority distributed channel congestion control approach based on IEEE $802.11 \mathrm{p}$. The object of this approach is to ensure the low collision rate and maximum transmission probability of the high priority information.

In the study of LTE ${ }^{[38]}$, Mosyagin et al. ${ }^{[39]}$ proposed an approach of vehicle access network based on $4 \mathrm{G}$ and LTE-A (LTE-Advanced). The test results reveal that the approach can be operated in the vehicle with the speed of $140 \mathrm{~km} / \mathrm{h}$. Araniti et al. ${ }^{[40]}$ summarized and analyzed the application of LTE system in the vehicular system. The authors first summarized the related research on LTE research institutions and industry. Then, they discussed the challenges in the current study about this problem, and predicted the development direction of LTE in IoV. Thota et al. ${ }^{[41]}$ predominantly concentrated on the establishment of communication architecture and model in the LTE-A system. Moreover, they provided the performance difference between 2D and 3D channels. Moreover, HUAWEI launched LTE-V (LTE-Vehicle) for warning and controlling vehicle collision ${ }^{1}$.

Based on the LTE and WLAN, mobile Internet can provide communication between vehicle and vehicle and between vehicle and network. LTE is 
the third mobile generation communication standard developed by the partnership project organization. The architecture of $\mathrm{LTE}^{[38]}$ is more flat simple, and it can reduce network nodes and complex system degree, which reduces the system delay. Moreover, it reduces the cost of network deployment and maintenance ${ }^{[42,43]}$. WiMAX (Worldwide interoperability for Microwave Access) ${ }^{[44]}$ and WLAN (Wireless Local Area Network) are another two wireless communication technologies based on IEEE 802.11. Since these types of wireless access technologies are complementary, considerable studies combine the two technologies to help the vehicle connect network. Yang et al. ${ }^{[4]}$ analyzed the possibility of combining WiMAX and LTE-A, and compared the throughput and delay of Vehicle-toInfrastructure (V2I) using two technologies. It has been proved that the technology can enhance the communication efficiency of V2I using the combination of WiMAX and WLAN. A hybrid of WiMAX and WLAN, named Carlink ${ }^{[45]}$, can provide the standards of vehicles communication and security of navigation systems.

\subsection{Network transport}

In IoV, vehicles and infrastructures access network using variety of wireless access technologies. However, there exist large differences between different technologies. Hence, a transmission and control network is required for shielding these differences, which imply that the heterogeneous networks integration is inevitable with the development of IoV. To realize the transmission control network, the heterogeneous networks should be integrated with high degree, which will at-tract many challenges. Hence, the integration of heterogeneous networks has become a hot research field.

SDN (Software Defined Network) can control network traffic flexibly through separating the network device control and data. In SDN, as a pipeline, network becomes more intelligent, and it can realize the network transmission and control. According to the differences produced in processing the vehicular data using different communication technologies (such as cellular network and DSRC), Cao et al. ${ }^{[46]}$ proposed an approach to shield these differences. He et al. ${ }^{[47]}$ proposed a novel vehicle communication architecture based on SDN. In this architecture, the differences of different heterogeneous access technologies can be shielded via SDN interchange interface. To eliminate the differences of cellular network and broadcast network, Keller et al. ${ }^{[48]}$ proposed a multi-radio network integration approach based on content distribution network. This integration network can satisfy services of audio and video. Cloud computing has the advantages of powerful computing, dynamic scheduling of the resource, providing on-demand services, processing massive information efficiently, and integrating management mechanisms. These advantages can be used for solving the problems of information sharing and transmission delay in IoV. Hence, combining the cloud and vehicle is significantly important for the development of $\mathrm{IoV}^{[49-53]}$. Vehicloud ${ }^{[51]}$, which is an architecture based on cloud computing, and solves vehicle communication instability problems via transferring the traditional vehicular network to service-based architecture. Abid et al. ${ }^{[52]}$ proposed a novel architecture combined vehicle with cloud computing, named VCloud, for solving the communication deficiencies problem of $\mathrm{V} 2 \mathrm{~V}$ and $\mathrm{V} 2 \mathrm{I}$ for current $3 \mathrm{G} / 4 \mathrm{G}$.

MEC (Mobile Edge Computing) integrates the Internet and wireless network effectively, and it adds the functions of computing, storage, and data processing in wireless network. Additionally, it builds an open platform for implant applications, and opens the information interaction between wireless networks and service servers via wireless application interface. MEC integrates the wireless networks and services, and it upgrades the traditional base station to intelligent base station. For the future network transmission and control, MEC will also play an important role. In addition, with the advent of the $5 \mathrm{G}$ era, the communication delay will be greatly reduced, and the road information will be promptly transmitted to the data platform. Hence, the platform can control the traffic more accurately and im- 
plement V2X communication applications.

All the above-mentioned technologies do not consider the vehicle feature (such as vehicle speed and bandwidth) for vehicle access and transport, which are required in $\mathrm{IoV}$ and important for vehicles to connect networks.

\section{Coordination computing control layer}

The coordination computation control layer is used for realizing computing and control on human, vehicle, and environment coordinately. It is a basis for realizing coordinated control and management. It can control individual and swarm via computing individual information coordinately. Furthermore, it can complete some traffic task in IoV. This layer can achieve the sustainability of service requirements via computing and control on-demand resource by scheduling coordinately.

\subsection{Coordination model of IoV object}

According to different demands from IoV, we can divide the coordination model into two categories from the perspective of microcosmic and macroscopic, that is, individual coordination model and swarm coordination model ${ }^{[54]}$.

The individual model is used for solving the coordination problems between human and vehicle, and individual object and swarm object. In this model, the individual object of human and vehicle consists of human (driver) and the driving car. The swarm object consists of all objects of IoV except the individual object. Human and vehicles interaction realizes the tight coupling and completes the direct interaction via in-vehicle network. In order to solve the bottleneck of communication, an intelligent image is required for acting as an agent for the intelligence of driver and vehicle. The agent of driver and vehicle can complete the objects coordination via analysis of the individual behavior.

The swarm model is used for solving the swarm coordination problem among human, vehicle, and environment in IoV from the perspective of cooperation services. In this model, human, vehicle, and environment coordinate with each other. The human includes human in vehicle (driver, passenger) and human in environment (rider, pedestrian). The vehicle includes driving vehicles and parking vehicles. Similarly, the environment includes filling/charging piles, environmental monitoring, and information channel services access points. Owing to the nonsymmetry of computing abilities among human, vehicle, and environment, the panoramic sensing of IoV not only relies on the intelligent vehicles, but also on the swarm coordination sensing. Human and vehicles perform an active part in the coordination computing via mobile phones and vehicles. However, they participate passivity through intelligent electronic stations, which is deployed by ITS. Therefore, the swarm coordination model of IoV consists of human, vehicles, environment, intelligent images, and services. In order to cope with the challenges of IoV, the intelligence of human and vehicles should be modeled in cyber space.

\subsection{Coordination computing control}

With the rapid development of wireless communication technology, more vehicle and user information can be collected via in-vehicle, V2V, and V2I communication mechanisms. Coordination computing can collect more effective information, such as vehicle states, road conditions, traffic conditions, and user habits, for establishing a vehicle-road coordination system, which can enhance the efficiency ${ }^{[16,55]}$, safety $^{[56]}$, and sustainability ${ }^{[57,58]}$ of the transportation system ${ }^{[59-61]}$.

Naboulsi et al. ${ }^{[55]}$ used the vehicle and road to coordinately perceive the city traffic data and computed the traffic to obtain the topology of city traffic network and its characteristics, upon which better IoV services can be provided. Lee et al. ${ }^{[59]}$ transferred the vehicles collaboration problem to the vehicle speed coordination problem by computing whether the vehicle trajectory intersects or not. Vehicles can coordinate at the intersection by solving 
the coordination problem. Ghaffarian et al. ${ }^{[56]}$ transformed the traffic problem into integer programming problem by analyzing the two-way single lane intersection structure. They perfectly solved the coordination problem with no signal traffic lights intersection environment, and reduced the average delay effectively. Milans et al. ${ }^{[60]}$ proposed an approach based on V2V and V2I to coordinate computing. In this approach, driverless vehicles with several different communication standards and different system structures can communicate with each other, and they make decisions in accordance with data exchange.

For the present research, they predominantly focus on the vehicles coordination, particularly in VANET. However, the IoV service also requires communication resource, computation resource, and data to coordinate in IoV. Moreover, there are only few studies that focus on human-vehicle-environment coordination. In this paper, we find that VVs in this layer can solve the coordination computing problem, which is explained in detail in section 7 .

\section{Application layer}

\subsection{Closed services}

Closed services are services that aim at specific industries or platforms, particularly services highly correlated with vehicles and transportation themselves. Driving safety is one of the predominant application indexes for vehicles and transportation, for example, active road safety can decrease the probability of traffic accidents and promote transportation safety. Based on shared information about positions of vehicles and intersections, speed and leading vehicle distance, the occurrence of a traffic accident can be predicted by vehicle to vehicle and vehicle to roadside base-stations communication. Drivers always react quickly to avoid traffic accidents. Most of the studies on active road safety applications predominantly concentrated on the intersection conflict forewarning, overtaking forewarning, collision forewarning, rearend forewarning, chain collision fore- warning, emergency vehicle forewarning, rescue assistance, emergency brake, traffic violation forewarning, traffic state notices, and so on ${ }^{[62]}$. Collision forewarning is one of the most concerned spots in security applications ${ }^{[63-65]}$. At present, the collision forewarning techniques are independent program packages provided by original equipment manufacturers as the basis of systems ${ }^{[66]}$. They provide notices of traffic accidents, warning of road conditions (such as slippery pavement), and rearend vehicles. In order to avoid a hybrid collision, Colombo et al. ${ }^{[67]}$ indicated a method, which utilizes the vehicles dynamic model to solve the vehicle scheduling problems.

Most applications in ITS are closed services. ITS is a complex distributed system combined with advanced technologies in areas of communication, sensing, mobile positioning, databases, intelligent information processing, and automatic control. ITS predominantly contains six basic subsystems. They are advanced city management system, advanced navigation system, advanced vehicle control system, business vehicle management, advanced public transportation system, and advanced urban transportation system ${ }^{[68]}$. The intelligent traffic management also includes ETC, which ensures vehicles to pass road and bridge toll stations at normal speed for reducing the probability of congestion ${ }^{[69-71]}$. In view of computing resources and storage resources caused by large-scale mobile users, a novel urban traffic management system is proposed for solving the problem of insufficient computing and storage resources based on intelligent transportation cloud ${ }^{[72]}$.

\subsection{Open services}

Open services in IoV are mainly provided by the third party for users, which are classified into online and offline stream media and human-machine interactive services, including video conference, weather information, data transmission, web services, music downloads, game interactivity, and roadside services. Future mobile internet services are extended to vehicular services, which provide services for vehicles, such as Apples CarPlay. Generally, the ser- 
vices provided for users include two aspects, namely personalized entertainment services and transportation services. Users entertainment services predominantly focus on those that can be obtained from network or other vehicles. For example, notice of points of interest, local ecommerce, and media downloads. However, the focus of personalized transportation services is predominantly focused on transportation data that the users should retrieve from networks, such as path navigation and HD (High Definition) maps for automated driving ${ }^{[73]}$.

Telematics also contains some open services ${ }^{[74]}$. Moreover, one of the typical open services is Telematics in taxis, which can collect trajectory data, analyze traffic state, and provide opening services. Telematics is a composite term of telecommunication and informatics. Moreover, it is a service system, which provides information through Internet technology, namely vehicular computer systems, wireless communication technology, satellite navigation devices, exchange texts, and voices ${ }^{[66]}$. Telematics provides functions about security applications, emergency rescue, guard against theft, and remote diagnosis. Telematics can contact with service center through wireless communication to locate the fault accurately, provide the fault causes and diagnosis to maintain personnel, and ensure the vehicles travel more safely. Utilizing cloud services, Telematics synchronizes data with other electronic devices, provides real-time road condition information, and selects the best route ${ }^{[75]}$. Telematics can update the latest map information to maintain the map data accurate and up-to-date. Then, it can query the information about surrounding facilities, parking plots, shops, and services. It can also provide the functions of telephone operators and one-touch calling center to reduce operation and facilitate users. Drivers can view weather, read news, browse fresh advices, download music and video, and play online games through Telematics, which makes driving more colorful. Meanwhile, with the rapid development of mobile communication and convergence of cellular and private networks, speed and traffic is no longer the bottleneck of the development of Telematics.
All the above-mentioned services focus on enhancing the driving safety and some common applications. Few of them combine global traffic information and the driver preference to provide personalized service, which could become more and more important in future. Thus, we should explore novel IoV service.

\section{IoV coordination computing based on VVs}

At present, the studies of IoV predominantly focus on micro and macro levels. At the micro level, the research focuses on intelligent vehicle driving and driving safety, which makes micro decisions based on the surrounding environment. These decisions invariantly do not consider the impact of traffic. Therefore, it cannot solve the existing traffic problems effectively. From the macro level, the research focuses on the procedure to guide and control vehicle flow, which makes macro decisions based on the global traffic information and the current traffic state. However, the macro decisions do not consider the personalized behavior of vehicle. Moreover, the solution invariantly indicates inefficiency when it is used for solving the traffic problem. Moreover, because the traffic prediction in macro studies depends on the historical traffic flow, the results are not accurate.

To eliminate the gaps from the middle studies level in IoV, we propose to configure an image that has intelligence of vehicle and driver in the cyber space, named Virtual Vehicle. VVs can connect individual vehicle control and vehicle flow control from the middle level, and realize the human-vehicle-environment coordination control. A VV is the image in cyber space (such as cloud) of the human and vehicle in physical space. This image includes the features and characteristics of human and vehicle. VV embodies the microcosmic behavioral features of driver and vehicle during the driving. Moreover, VVs can interact directly with each other in cyber space by providing traffic service and sharing sensing data coordinately, 


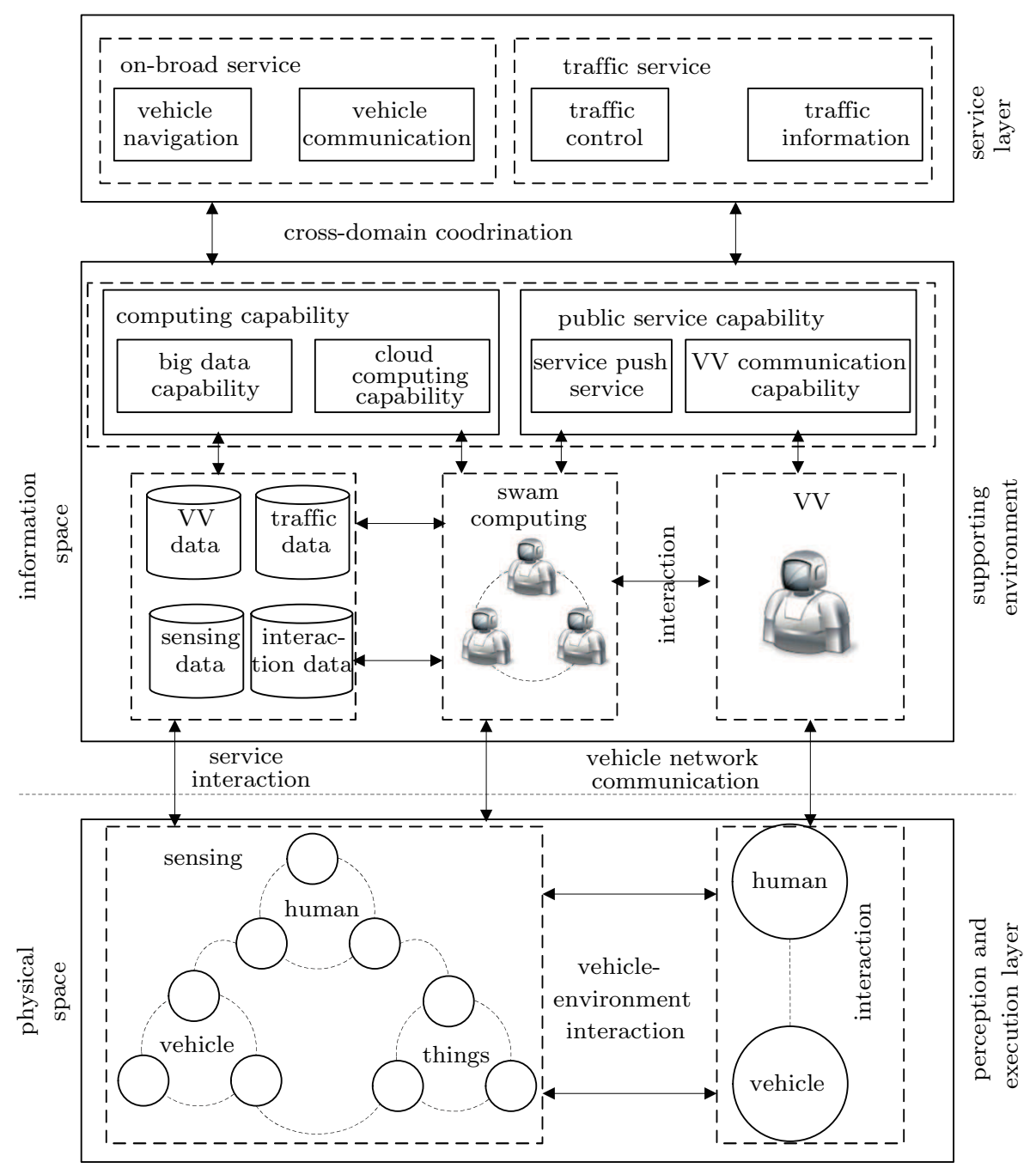

Figure 2 Architecture of IoV

which can solve the bottleneck of communication in physical space.

A VV combines the driverless vehicle and ITS from the middle perspective. Moreover, we provide the architecture of a $\mathrm{VV}$ as shown in Fig. 2. The architecture includes the perception and execution layer in physical space and the supporting environment and service layer in cyber space. The perception and execution layer provides the ability of sensing and control for human, vehicle, and environment in physical space. From the figure, same driver in different vehicles can compose different VVs, and same vehicle with different vehicles can also compose different VVs. In this layer, the driver-vehicle cognition model should be constructed accordingly to sense the driving behavior and vehicle characteristic. This model not only senses the information of driver and vehicle, but also recognizes the differences of driving behavior with different drivers. Based on the differences, we can obtain the personalization characteristic for a $\mathrm{VV}$, which provides the personal services and the basis of swarm coordination control.

The supporting environment, which is provided by coordination computing control layer, provides a platform for VV operation, and computing capability and public service capability. A VV interacts with the corresponding vehicle through the vehicle to network communication, which can realize the coordination between a VV and driverless vehicle. Simultaneously, VVs can interact with each other in 
the environment. Therefore, they can realize the swarm intelligence computation. In addition, it is essential for the supporting environment to deal with the traffic data from ITS, the sensing and interaction data by itself, which are the basis for a VV in decision making and interaction. In supporting environment, the computing capability and public service capability can be provided for a VV to compete its computing and service function. The service layer includes on-board service and traffic service. On-board service includes vehicle navigation service, vehicle communication services, and vehicle entertainment services, such as Telematics. Traffic service predominantly provides traffic control, environment coordinated control, and push accurate traffic services to traffic control department or other transportation service providers. For the on-board service, a VV can provide personalized services according to the results of global traffic information and interaction with others. For traffic service, traffic control department can obtain the vehicle current state and predict future behavior accurately because of the existence of VVs in cyber space, which can be used for realizing the message broadcast and traffic control.

\section{Conclusion and future work}

In IoV, human, machine, vehicle, and environment can coordinate. Mobile Internet, ITS, cloud computing, automotive electronics, intelligent geographic information system are integrated via advanced information communication and processing technology. Hence, the industry chain of IoV includes the automotive manufacturing and services, communications and information services, financial and insurance services, and public service fields. This paper reviews the development of IoV from the perspective of architecture and key technology and summarizes the existing challenges in IoV. Then, this paper configures the VVs in IoV in cyber space and provides the nature and architecture of a VV. In future research, we will pay more attention to realizing VVs.

\section{References}

[1] W. Sun. Internet of Vehicles [J]. Advances in media technology, 2013: 47.

[2] H. M. Mora, D. Gil, J. F. C. Lpez, et al. Flexible framework for real-time embedded systems based on mobile cloud computing paradigm [J]. Mobile information systems, 2015: ID 652462.

[3] K. M. Alam, M. Saini, A. El Saddik. Toward social internet of vehicles: concept, architecture, and applications [J]. IEEE access, 2015, 3: 343-357.

[4] J. Markoff. Google cars drive themselves, in traffic [J]. The New York times, 2010, 10(A1): 9.

[5] A. Slywotzky, R. Wise. Demand innovation: GMs OnStar case [J]. Strategy \& leadership, 2003, 31(4): 17-22.

[6] H. Hartenstein, K. Laberteaux. VANET vehicular applications and inter-networking technologies [M]. Manhattan: John Wiley \& Sons, 2009.

[7] K. Li. Status and strategic recommendations of development on intelligent connected vehicle $[\mathrm{J}]$. Auto business review, 2016, (2): 170-175.

[8] J. L. Li, Z. H. Liu, F. C. Yang. Internet of Vehicles: the framework and key technology [J]. Journal of Beijing University of Posts \& Telecommunications, 2014, 37: 95-100.

[9] D. A. Johnson, M. M. Trivedi. Driving style recognition using a smartphone as a sensor platform [C]//The 14th International IEEE Conference on Intelligent Transportation Systems (ITSC), 2011: 1609-1615.

[10] C. Tran, M. M. Trivedi. Towards a vision-based system exploring 3D driver posture dynamics for driver assistance: Issues and possibilities [C]//Intelligent Vehicles Symposium (IV), 2010: 179-184.

[11] R. Baldessari, B. Bdekker, M. Deegener, et al. Car-2car communication consortium-manifesto [J]. Car 2 car communication consortium, 2007.

[12] E. Guizzo. How googles self-driving car works [J]. IEEE spectrum online, 2011.

[13] H. Zhou, H. Kong, L. Wei. Efficient road detection and tracking for unmanned aerial vehicle [J]. IEEE transactions on intelligent transportation systems, 2015, 16 (1): 297-309.

[14] J. M. Á. Alvarez, A. M. Lopez. Road detection based on illuminant invariance $[\mathrm{J}]$. IEEE transactions on intelligent transportation systems, 2011, 12 (1): 184-193.

[15] B. S. Kerner, H. Rehborn, R. P. Schfer, et al. Traffic dynamics in empirical probe vehicle data studied with three-phase theory: spatiotemporal reconstruction of traffic phases and generation of jam warning messages [J]. Physica a: statistical mechanics and its applications, 2013, 392 (1): 221-251.

[16] S. Santini, A. Salvi, A. Valente, et al. A consensus-based approach for platooning with inter-vehicular communications [C]//IEEE Conference on Computer Communications (INFOCOM), 2015: 1158-1166. 
[17] R. Siegwart, I. R. Nourbakhsh, D. Scaramuzza. Introduction to autonomous mobile robots $[\mathrm{M}]$. Cambridge: MIT press, 2011.

[18] A. L. Meyrowitz, D. R. Blidberg, R. C. Michelson. Autonomous vehicles [J], Proceedings of the IEEE, 1996, 84 (8): 1147-1164.

[19] C. Urmson, J. Anhalt, D. Bagnell, et al. Autonomous driving in urban environments: boss and the urban challenge [J]. Journal of field robotics. 2008, 25 (8) : 425466.

[20] D. Ferguson, T. M. Howard, M. Likhachev. Motion planning in urban environments: part ii [C]//IEEE/RSJ International Conference on Intelligent Robots and Systems, 2008: 1070-1076

[21] M. Slosson, C. Johnston, P. Barbara. Google gets first self-driven car license in Nevada [EB/OL]. https://www.dawn.com/news/716695/google-gets-firstself-driven-car-license-in-nevada, 2012.

[22] P. Falcone, F. Borrelli, J. Asgari, et al. Predictive active steering control for autonomous vehicle systems $[\mathrm{J}]$. IEEE transactions on control systems technology, 2007, 15 (3): 566-580.

[23] D. W. Whitcomb, W. F. Milliken. Design implications of a general theory of automobile stability and control $[\mathrm{J}]$. Proceedings of the institution of mechanical engineers: automobile division, 1956, 10: 367-425.

[24] L. Segel. Theoretical prediction and experimental substan-tiation of the response of the automobile to steering control $[\mathrm{J}]$. Proceedings of the institution of mechanical engineers: automo-bile division, 1956: 310-330.

[25] J. Hedrick, M. Tomizuka, P. Varaiya. Control issues in automated highway systems [J]. IEEE control systems, 1994, 14(6): 21-32.

[26] L. Li, F. Y. Wang, Y. Zhang. Research and developments of intelligent driving behavior analysis $[\mathrm{J}]$. Acta automatica sinica, 2007, 33 (10): 1014-1022.

[27] J. Wang, D. Zhang, J. Liu, et al. V2I-based multiobjective driver assistance system for intersection support [C]//International IEEE conference on intelligent transportation systems, 2010: 348-353.

[28] J. C. McCall, M. M. Trivedi. Video-based lane estimation and tracking for driver assistance: survey, system, and evaluation [J]. IEEE transactions on intelligent transportation systems, 2006, 7 (1): 20-37.

[29] J. Hilgert, K. Hirsch, T. Bertram, et al. Emergency path planning for autonomous vehicles using elastic band theory [C]//IEEE/ASME International Conference Advanced Intelligent Mechatronics, 2003: 1390-1395.

[30] L. Fletcher, L. Petersson, A. Zelinsky. Road scene monotony detection in a fatigue management driver assistance system [C]//Intelligent Vehicles Symposium, 2005: 484-489.

[31] D. T. McRuer, L. Hofmann, H. Jex, et al. New approaches to human-pilot/vehicle dynamic analysis [M]. Hawthorne: Systems Technology, Inc., 1968.
[32] D. Jiang, V. Taliwal, A. Meier, et al. Design of $5.9 \mathrm{GHz}$ DSRC-based vehicular safety communication [J]. IEEE wireless communications, 2006, 13 (5): 36-43.

[33] U. Lee, M. Gerla. A survey of urban vehicular sensing platforms [J]. Computer networks, 2010, 54 (4): 527544.

[34] M. Kakkasageri, S. Manvi. Information management in vehicular Ad hoc networks: a review [J]. Journal of network and computer applications, 2014, 39 (1): 334-350.

[35] V. Cevher, R. Chellappa, J. H. McClellan. Vehicle speed estimation using acoustic wave patterns [J]. IEEE transactions on signal processing, 2009, 57 (1): 30-47.

[36] C. W. Hsu, C. H. Hsu, H. R. Tseng. MAC channel con-gestion control mechanism in IEEE 802.11 p/WAVE vehicle networks $[\mathrm{C}] / /$ Vehicular Technology Conference (VTC Fall), 2011: 1-5.

[37] X. Shen, X. Cheng, R. Zhang, et al. Distributed congestion control approaches for the IEEE 802.11 p vehicular networks [J]. IEEE intelligent transportation systems magazine, 2013, 5 (4): 50-61.

[38] S. Sesia, M. Baker, I. Toufik. LTE-the UMTS long term evolution: from theory to practice [M]. Manhattan: John Wiley \& Sons, 2011.

[39] J. Mosyagin. Using 4G wireless technology in the car [C]//International Conference on Transparent Optical Networks, 2010: 1-4.

[40] G. Araniti, C. Campolo, M. Condoluci, et al. LTE for vehicular networking: a survey [J]. IEEE communications magazine, 2013, 51 (5): 148-157.

[41] J. Thota, R. Almesaeed, A. Doufexi, et al. Infrastructure to vehicle throughput performance in LTE-A using 2D and 3D 3GPP/ITU channel models [C]//Vehicular Technology Conference (VTC Spring), 2015: 1-5.

[42] T. Cai, G. P. Koudouridis, C. Qvarfordt, et al. Coverage and capacity optimization in EUTRAN based on central coordination and distributed Gibbs sampling [C]//Vehicular Technology Conference (VTC 2010Spring), 2010: 1-5.

[43] L. Militano, M. Condoluci, G. Araniti, et al. Bargaining solutions for multicast subgroup formation in LTE [C]//Vehicular Technology Conference (VTC Fall), 2012: 1-5.

[44] Y. Yang, H. Hu, J. Xu, et al. Relay technologies for WiMAX and LTE-advanced mobile systems [J]. IEEE communications magazine, 2009, 47 (10): 100-105.

[45] T. Sukuvaara, C. Pomalaza-Rez. Vehicular networking pilot system for vehicle-to-infrastructure and vehicleto-vehicle communications [J]. International journal of communication networks and information security, 2009, 1(3): 1-11.

[46] Y. Cao, J. Guo, Y. Wu. SDN enabled content distribution in vehicular networks [C]//International Conference on Innovative Computing Technology (INTECH), 2014: 164-169.

[47] Z. He, J. Cao, X. Liu. SDVN: enabling rapid network 
innovation for heterogeneous vehicular communication [J]. IEEE network, 2016, 30 (4): 10-15.

[48] R. Keller, T. Lohmar, R. Tonjes, et al. Convergence of cellular and broadcast networks from a multi-radio perspective [J]. IEEE personal communications,2001, 8 (2): $51-56$.

[49] L. Zeng, Q. Han, X. Wu, et al. Abnormal vehicle oriented infrastructure based on wireless sensor network and cloud computing [J]. Sensor letters, 2013, 11 (5): 918-925.

[50] M. Gerla. Vehicular cloud computing [C]//Annual Mediterranean on Ad hoc Networking Workshop (MedHoc-Net), 2012: 152-155.

[51] Y. Qin, D. Huang, X. Zhang. Vehicloud: cloud computing facilitating routing in vehicular networks [C]//International Conference on Trust, Security and Privacy in Computing and Communications, 2012: 1438-1445.

[52] H. Abid, L. T. T. Phuong, J. Wang, et al. V-Cloud: vehicular cyber-physical systems and cloud computing [C]//International Symposium on Applied Sciences in Biomedical and Communication Technologies, 2011,165.

[53] J. Wan, D. Zhang, Y. Sun, et al. VCMIA: a novel architecture for integrating vehicular cyber-physical sys-tems and mobile cloud computing [J]. Mobile networks and applications, 2014, 19 (2): 153-160.

[54] F. C. Yang, S. G. Wang, J. L. Li, et al. An overview of internet of vehicles [J]. China communications, 2014, 11 (10): 1-15.

[55] D. Naboulsi and M. Fiore. On the instantaneous topology of a large-scale urban vehicular network: the cologne case $[\mathrm{C}] / /$ ACM International Symposium on Mobile Ad hoc Networking and Computing, 2013: 167-176.

[56] H. Ghaffarian, M. Fathy, M. Soryani. Vehicular Ad hoc networks enabled traffic controller for removing traffic lights in isolated intersections based on integer linear programming $[\mathrm{J}]$. IET intelligent transport systems, 2012, 6 (2): 115-123.

[57] C. Wang, J. Li, F. Ye, et al. Multi-vehicle coordination for wireless energy replenishment in sensor networks [C]//International Symposium on Parallel \& Distributed Processing (IPDPS), 2013: 1101-1111.

[58] J. H. Liu, J. M. Sun, J. P. Bi, et al. VANET cooperative downloading approach study based on dynamic slot [J]. Jisuanji xuebao (Chinese journal of computers), 2011, 34 (8): 1378-1386.

[59] J. Lee, B. Park. Development and evaluation of a cooperative vehicle intersection control algorithm under the connected vehicles environment [J]. IEEE transactions on intelligent transportation systems, 2012, 13 (1): 8190.

[60] V. Milans, J. Alonso, L. Bouraoui, et al. Cooperative maneuvering in close environments among cybercars and dualmode cars $[\mathrm{J}]$. IEEE transactions on intelligent transportation systems, 2011, 12 (1): 15-24.
[61] R. Fei, K. Yang, X. Cheng. A cooperative social and vehic-ular network and its dynamic bandwidth allocation algorithms $[\mathrm{C}] / /$ IEEE Conference on Computer Communications Workshops (INFOCOM WKSHPS), 2011: 63-67

[62] G. Vivo, P. Dalmasso, F. Vernacchia. The European Integrated Project" SAFESPOT"-How ADAS applications cooperate for the driving safety [C]//IEEE Intelligent Transportation Systems Conference, 2007: 624-629.

[63] V. Desaraju, H. C. Ro, M. Yang, et al. Partial order techniques for vehicle collision avoidance: application to an autonomous roundabout testbed [C]//IEEE International Conference on Robotics and Automation, 2009: $82-87$.

[64] J. M. Duperret, M. R. Hafner, D. D. Vecchio. Formal design of a provably safe robotic roundabout system [C]//IEEE/RSJ International Conference on Intelligent Robots and Systems (IROS), 2010: 2006-2011.

[65] M. Hafner, D. Cunningham, L. Caminiti, et al. Automated vehicle-to-vehicle collision avoidance at intersections $[\mathrm{C}] / /$ World Congress on Intelligent Transport Systems, 2011.

[66] E. Hossain, G. Chow, V. C. Leung, et al. Vehicular telematics over heteroge-neous wireless networks: a survey [J]. Computer communications, 2010, 33 (7): 775793.

[67] A. Colombo, D. D. Vecchio. Efficient algorithms for collision avoidance at intersections [C]//ACM International Conference on Hybrid Systems: Computation and Control, 2012: 145-154.

[68] J. S. Sussman. Perspectives on intelligent transportation systems (ITS) [M]. Dordrecht: Springer Science \& Business Media, 2008.

[69] L. Li, F. Y. Wang. Cooperative driving at blind crossings using intervehicle communication [J]. IEEE transactions on vehicular technology, 2006, 55 (6): 1712-1724.

[70] D. B. Rawat, C. Bajracharya, G. Yan. Towards intelligent transportation cyber-physical systems: real-time computing and communications perspectives $[\mathrm{C}] / /$ IEEE SoutheastCon, 2015, 9 (3): 1-6.

[71] C. W. Chen, X. C. Chen, I. H. Peng, et al. Study of safety and efficient routing for intelligent transportation system [C]//Asian Himalayas International Conference on Internet, 2009: 1-5.

[72] Z. Li, C. Chen, K. Wang. Cloud computing for agentbased urban transportation systems [J]. IEEE intelligent systems, 2011, 26 (26): 73-79.

[73] G. Karagiannis, O. Altintas, E. Ekici, et al. Vehicular networking: a survey and tutorial on re-quirements, architectures, challenges, standards and solutions $[\mathrm{J}]$. IEEE communications surveys \& tutorials, 2011, 13 (4): 584-616.

[74] P. Nijkamp, G. Pepping, D. Banister. Telematics and transport behaviour $[\mathrm{M}]$. Dordrecht: Springer Science \& Business Media, 2012. 
[75] M. B. Phalake, D. M. Bhalerao. Vehicle telematics system using GPRS $[\mathrm{J}]$. International journal of computer applications in technology, 2011, 2(1): 132-135.

\section{About the authors}

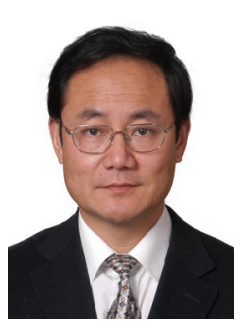

Fangchun Yang received his Ph.D. degree in communications and electronic systems from Beijing University of Posts and Telecommunications (BUPT), China. He is currently a professor at the State Key Laboratory of Networking and Switching Technology, BUPT. He is a fellow of the IET. His current research interests include network intelligence, service computing, Internet of Vehicle. (Email: fcyang@bupt.edu.cn)

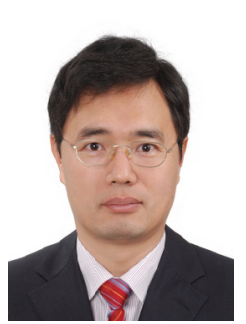

Jinglin Li [corresponding author] received his Ph.D. degree in computer science and technology from Beijing University of Posts and Telecommunications (BUPT), China. He is currently an associate professor at the State Key Laboratory of Networking and Switching Technology, BUPT. His research interests are mainly in the areas of network intelligence, mobile Internet, Internet of Things, Internet of Vehicles. (Email: jlli@bupt.edu.cn)

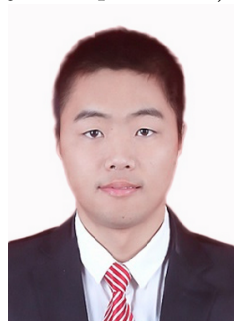

Tao Lei received his M.E. in computer science and technology from North China University of Water Resources and Electric Power, China. He is currently a Ph.D. candidate at Beijing University of Posts and Telecommunications (BUPT). His research interests include mobile computing, multiagent system, Internet of Vehicle. (Email: leitao@bupt.edu.cn)

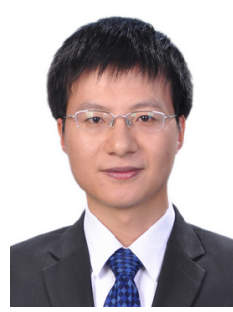

Shangguang Wang received his Ph.D. degree in computer science and technology from Beijing University of Posts and Telecommunications (BUPT), China. He is currently associate professor at the State Key Laboratory of Networking and Switching Technology, BUPT. He is a senior member of the IEEE., vice chair of IEEE Computer Society Technical Committee on Services Computing, president of the Service Society Young Scientist Forum in China. His research interests include IoV, service computing, cloud computing, and QoS management. (Email: sgwang@bupt.edu.cn) 